\title{
Angiographic Structural Differentiation between Native Arteriogenesis and Therapeutic Synangiosis in Intracranial Arterial Steno-Occlusive Disease
}

\author{
(D).C. Ooi, (D)A.N. Laiwalla, (D) R. Liou, and (D).R. Gonzalez
}

\begin{abstract}
BACKGROUND AND PURPOSE: Encephaloduroarteriosynangiosis has been shown to generate collateral vessels from the extracranialto-intracranial circulation in patients with Moyamoya disease and intracranial arterial steno-occlusive disease. The mechanisms involved are not well-understood. We hypothesized that angiogenesis is the leading mechanism forming collaterals after encephaloduroarteriosynangiosis because there are no pre-existing connections. Angiogenesis-generated collaterals should exhibit higher architectural complexity compared with innate collaterals.
\end{abstract}

MATERIALS AND METHODS: Pre- and postoperative digital subtraction angiograms were analyzed in patients enrolled in a prospective trial of encephaloduroarteriosynangiosis surgery. Branching angioscore, tortuosity index, and local connected fractal dimension were compared between innate and postoperative collaterals.

RESULTS: One hundred one angiograms (50 preoperative, 51 postoperative) were analyzed from 44 patients (22 with intracranial atherosclerosis and 22 with Moyamoya disease). There was a significantly higher median branching angioscore (13 versus $4, P<.001)$ and a lower median tortuosity index (1.08 versus $1.76, P<.001)$ in the encephaloduroarteriosynangiosis collaterals compared with innate collaterals. Higher mean local fractal dimension peaks $(1.28 \pm 0.1$ versus $1.16 \pm 0.11, P<.001)$ were observed in the encephaloduroarteriosynangiosis collaterals compared with innate collaterals for both intracranial atherosclerosis $(P<.001)$ and Moyamoya disease $(P<.001)$ groups. The observed increase in high connectivity was greater in the intracranial atherosclerosis group compared with patients with Moyamoya disease $(P=.01)$.

CONCLUSIONS: The higher median branching angioscore and local connected fractal dimension, along with the lower median tortuosity index of encephaloduroarteriosynangiosis collaterals, are consistent with the greater complexity observed in the process of sprouting and splitting associated with angiogenesis.

ABBREVIATIONS: EDAS = encephaloduroarteriosynangiosis; ICAS = intracranial atherosclerosis; ICASD = intracranial arterial steno-occlusive disease; LCFD = local connected fractal dimension; MMD = Moyamoya disease

ntracranial arterial steno-occlusive disease (ICASD) is one of the most common vascular abnormalities found worldwide in patients with acute ischemic stroke. ${ }^{1}$ ICASD accounts for $10 \%$ of strokes in whites and as much as $67 \%$ of strokes in Asian, His-

Received June 30, 2015; accepted after revision December 1.

From the Departments of Neurosurgery (Y.C.O., A.N.L., R.L., N.R.G.) and Radiology (N.R.G.), David Geffen School of Medicine at the University of California, Los Angeles, Los Angeles, California.

This work was supported by the Ruth and Raymond Stotter Endowed Chair in Neurosurgery and the National Institutes of Health-National Institute of Neurological Disorders and Stroke award, K23NS079477.

The authors have no personal, financial, or institutional interest in any of the drugs, materials, or devices described in this article.

Paper previously presented in part at: Annual Scientific Meeting of the American Association of Neurological Surgeons, May 2-6, 2015; Washington, DC; and Annual Meeting of the California Association of Neurological Surgeons, January 16-18, 2015; Newport Beach, California. panic, and black patients. ${ }^{1}$ ICASD carries a worse prognosis than other stroke etiologies, with a rate of recurrent stroke and death between $15 \%$ and $25 \%$ per year despite maximal medical therapy. ${ }^{2}$ While patients with ICASD develop spontaneous collaterals to areas of ischemia, these alternative conduits fail with time and lead to a progression of their symptoms, resulting in transient ischemic attacks, strokes, or even death. ${ }^{3}$ Indirect cerebral revascularization via encephaloduroarteriosynangiosis (EDAS) has been successful in establishing collateral flow in several forms of ICASD, including Moyamoya disease (MMD) and intracranial atherosclerosis (ICAS). ${ }^{4,5}$ However, the mechanisms involved in

Please address correspondence to Nestor R. Gonzalez, MD, David Geffen School of Medicine at UCLA, 300 Stein Plaza, Suite 562, Los Angeles, CA 90095; e-mail: ngonzalez@mednet.ucla.edu

- Indicates open access to non-subscribers at www.ajnr.org

http://dx.doi.org/10.3174/ajnr.A4675 
innate collateral formation and neovascularization induced by EDAS are not fully understood.

Innate collateral vessels that occur spontaneously in ICASD are formed by arteriogenesis. ${ }^{6}$ The formation of collaterals via arteriogenesis is driven by sheer stress and involves the recruitment and enlargement of preexisting vessels in a flow-dependent hypoxia-independent process. ${ }^{6}$ Conversely, due to the lack of preexisting collateral vessels between the portions of the external carotid artery used during EDAS and the ICA, formation of EDAS should be primarily driven by angiogenesis. ${ }^{7}$ Angiogenesis is induced by hypoxia and involves the formation of new collateral vessels via sprouting or splitting from preexisting vascular structures, resulting in collaterals of greater complexity.

A better understanding of the differences in morphology and clinical implications of these features between spontaneously occurring collaterals in ICASD and new collaterals formed after EDAS may provide valuable insight into ICASD and its treatment. We designed an angiography-based study by using patients from a prospective trial of EDAS surgery to test the hypothesis that neovascularization generated after EDAS has greater angioarchitectural complexity compared with spontaneously formed innate collaterals in ICASD. To quantify the complexity of the vascular trees, we measured branching pattern, tortuosity, and fractal connectivity.

\section{MATERIALS AND METHODS}

Pre- and postoperative digital subtraction angiograms were analyzed in patients enrolled in a prospective trial of EDAS surgery (Surgical Indirect Revascularization for Symptomatic Intracranial Arterial Stenosis [ERSIAS] trial; clinicaltrials.gov identifier: NCT01819597). The study was conducted with institutional review board approval. All patients enrolled in the study had experienced either a TIA or nonsevere stroke. All EDAS operations were performed by 1 of 2 senior vascular neurosurgeons. EDAS is a form of indirect revascularization or external carotid-internal carotid bypass. It involves the dissection and relocation of superficial temporal artery and/or middle meningeal artery branches. These arterial branches are separated from their surrounding tissues under microscopic visualization, rerouted through a craniotomy, and then placed intracranially in close proximity to branches of the MCA. The MCA branches are dissected in the arachnoid space, and the superficial temporal artery and/or middle meningeal artery branches are kept in position with microsutures to the arachnoid or dural cuffs, maintaining close contact between the external carotid and MCA branches. ${ }^{2,4,5}$ Preoperative angiograms were obtained within a week before the operation. Postoperative angiograms were obtained at the 3-, 6-, and 12month intervals postoperatively. The most recent postoperative angiogram available was used for this study. All angiograms were obtained by a senior neuroradiologist, by using standard lateral and posterior-anterior views at 3 frames per second. Selective ICA and external carotid artery injections were performed to minimize vessel overlap.

Innate collaterals for patients with ICAS were defined by first establishing the angiographic limits among the major branches of the anterior cerebral artery, middle cerebral artery, and posterior cerebral artery territories and then identifying leptomeningeal collateral vessels crossing these territories (Figs 1 and 2). For patients with MMD, "innate collaterals" were defined as deep collaterals with a Moyamoya-like appearance. "EDAS collaterals" were defined as new branches observed from the superficial temporal artery or middle meningeal artery that produced cerebral contrast blush and subsequently drained into cerebral veins. Selected collateral vessels were isolated by dynamic delineation for further analysis. With dynamic delineation, vessel flow was followed through the arterial phases of the catheter angiogram, establishing the continuity of vessels. These vessels were traced and marked, excluding any overlapping and/or underlying vessels. Delineated vessels were analyzed by 3 independent observers, including 2 neuroradiologists and 1 senior neuroradiologist. The interobserver agreement $(\kappa)$ was calculated. Marked vessels were converted to a binary black and white image (Fig 3). All image processing was performed by using ImageJ software (National Institutes of Health, Bethesda, Maryland). ${ }^{8}$

Angioarchitectural differences between innate and EDAS collaterals were evaluated by comparing the quantitative measures of branching pattern and tortuosity. Branching pattern was measured by using the branching angioscore (Fig 4). ${ }^{9}$ A 10,000-pixelper-box grid was overlaid on the delineated vessels. The branching angioscore was defined as the total number of branching points within a single box. This was measured in all boxes within the grid containing portions of the delineated vessel, and the box with the highest branching angioscore was selected. Tortuosity was measured with the artery tortuosity index, by using the longest branch of the delineated vessel between 2 branching points (Fig 5). ${ }^{10}$ The artery tortuosity index was calculated by the quotient of the actual vessel length and the straight-line distance of a delineated vessel between 2 branching points. Group indices were compared with the Wilcoxon rank sum test.

The local connected fractal dimension (LCFD) of delineated vessels was measured by using the Fraclac plugin for Image J. ${ }^{11}$ LCFD provides an index of complexity by measuring changes in connectivity with varying scales, allowing quantification of nonEuclidean geometric patterns. Fraclac performs LCFD analysis by selecting a seed pixel on the marked vessel and calculating the total number of pixels connected to the seed in a square area around the seed. The process is repeated for concentric squares of different sizes. On the basis of the rate of change of connected pixels within the different sizes of squares, it computes the fractal dimension for that pixel (Fig 6). This process is iterated over each pixel of the delineated vessel. High connectivity was defined as LCFD $\geq 1$.2. Log-transformations were used for skewed data. Comparison of means was performed by using a 2-tailed unpaired $t$ test for the aggregate group and a 2-tailed paired $t$ test for matched samples for MMD and ICAS. The Spearman correlation was used to test the associations among the variables.

\section{RESULTS}

The study population included 44 patients (4-84 years of age; mean, $35 \pm 19.2$ years), 30 females (68\%) and 14 males (32\%). Twenty-two patients had ICAS (7-84 years of age; mean, $49 \pm 16.9$ years) with stenosis in the intracranial ICA and/or MCA. Twenty-two patients had MMD (4-56 years of age; mean, $29 \pm 14.6$ years), with 13 patients (59\%) at Suzuki stage 3 and 9 

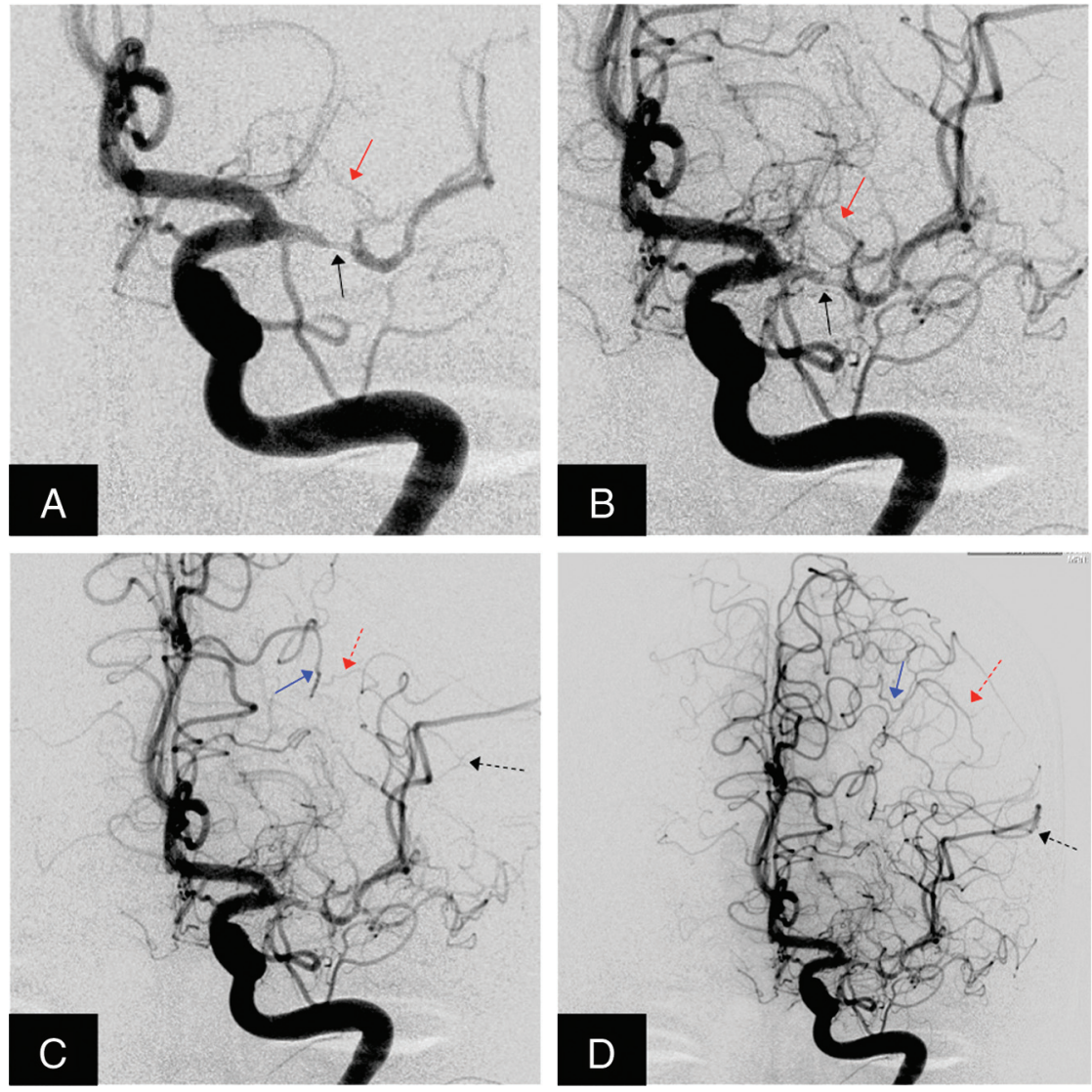

FIG 1. Cerebral angiogram anteroposterior views of the left internal carotid artery injection in a patient with intracranial atherosclerosis involving the middle cerebral artery that identify innate deep collaterals and leptomeningeal collaterals. Early arterial phase $(A)$ and late arterial phase $(B)$ show stenosis involving the middle cerebral artery (black arrow), with collaterals arising from the medial lenticulostriate arteries and connecting to the lateral lenticulostriate arteries, corresponding to deep collaterals providing flow to the MCA circulation. In the midarterial phase $(C)$ and late arterial phase $(D)$, normal branches of the anterior cerebral artery (blue arrow) are seen extending beyond the normal watershed territory. There is a delay in filling of the MCA branches (black dotted arrow). Leptomeningeal collaterals (red dotted arrow) are seen between the ACA and MCA branches.

patients $(41 \%)$ at Suzuki stage 4 . The mean age in the MMD group was significantly lower than that of the ICAS group $(P<$ $.001)$. There were 15 females $(68 \%)$ in both the ICAS and MMD groups. Seven patients required bilateral EDAS ( 6 females, 1 male). Of 102 angiograms (51 preoperative, 51 postoperative), 1 preoperative angiogram was excluded due to a lack of identifiable collaterals. The interobserver agreement $(\kappa)$ for delineated vessels in both pre- and postoperative angiograms was 0.813 .

\section{Branching Angioscore}

The branching angioscores were not normally distributed. There was a significantly higher median branching angioscore in the EDAS collaterals compared with the innate collaterals (13 versus $4, P<.001)$. This was significantly different for both the ICAS and MMD groups (ICAS: 11 versus $4, P<.001$; MMD: 15 versus 5 , $P<.001)$. The Table provides additional details of the branching angioscores.

\section{Tortuosity Index}

The tortuosity indices were not normally distributed. There was a significantly lower median tortuosity index in the EDAS collaterals compared with the innate collaterals (1.08 versus $1.76, P<$
.001). This was significantly different for both the ICAS and MMD groups (ICAS: 1.91 versus $1.08, P<.001$; MMD: 1.73 versus $1.09, P<.001)$.

\section{Local Connected Fractal Dimension}

The LCFDs were normally distributed.

There was a significantly higher mean LCFD in the EDAS collaterals compared with the innate collaterals $(1.28 \pm 0.1$ versus $1.16 \pm 0.11, P<.001)$. The proportion of high connectivity (LCFD $\geq$ 1.2) in the entire study population was significantly greater in the EDAS collaterals $(P<.001)$ than in the innate collaterals. In the ICAS group, the mean LCFD was significantly higher in the EDAS collaterals $(1.27 \pm 0.11)$ versus innate collaterals $(1.13 \pm 0.12, P<$ $.001)$. This relationship also held in the MMD group, with the mean LCFD in EDAS collaterals being $1.29 \pm 0.09$ versus $1.17 \pm 0.1$ in the innate collaterals $(P<.001)$. The proportion of high connectivity was also greater in the MMD group $(P<.001)$ and the ICAS group $(P<.001)$ separately. The Spearman correlation showed a strong association between LCFD peaks and both the branching angioscore $(P<.001)$ and the tortuosity index $(P<.001)$.

\section{DISCUSSION}

Indirect cerebral revascularization via EDAS has been shown to establish new collaterals through the development of vessels from the external carotid artery to the internal carotid artery in patients with ICASD. ${ }^{4,5}$ This phenomenon has been extensively described in the literature ${ }^{12-21}$; however, the mechanism involved in the formation of EDAS collaterals remains poorly understood. This is the first study to quantitatively compare the angioarchitecture of newly formed EDAS collaterals with that of existing innate collaterals in patients with ICASD, providing insight into the underlying mechanisms involved in collateral vessel formation.

Angiogenesis, primarily driven by hypoxia, involves the formation of new vessels through sprouting and splitting from preexisting vascular structures. ${ }^{7,22}$ Hypoxia regulates angiogenesis by activation of the hypoxia-inducible factor, which, in turn, modifies a variety of pro- and antiangiogenic factors, causing a shift toward an angiogenic phenotype. ${ }^{23-25}$ Animal studies performed by Luo et al, ${ }^{26}$ have shown that despite this shift toward a proangiogenic state, hypoxia ultimately leads to the inhibition of new vessel formation by brain endothelial cells. The failure to produce a neovascularization response has been attributed to the highly specialized nature of brain endothelial cells and the pleiotropic effects of the angiogenic factors produced by them. ${ }^{26}$ Native collaterals, however, occur via a process known as arteriogenesis, 

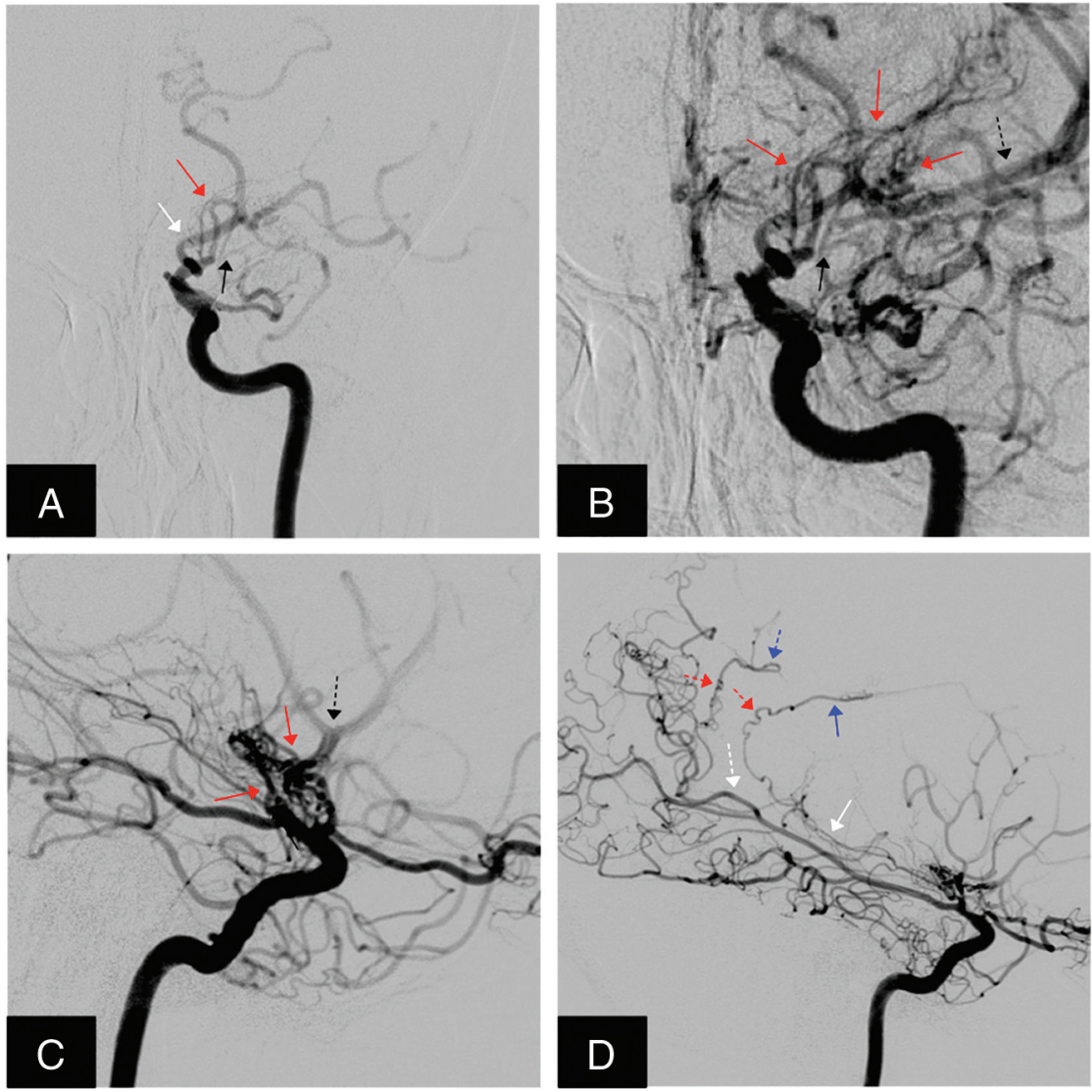

FIG 2. Cerebral angiogram anteroposterior $(A$ and $B)$ and lateral $(C$ and $D)$ views in a patient with Moyamoya disease with narrowing of the middle cerebral artery, identify innate deep collaterals and leptomeningeal collaterals. Early arterial phase $(A)$ and late arterial phase $(B)$ show normal posterior cerebral artery (white arrow), stenosis involving the middle cerebral artery (black arrow), with deep collaterals arising from the anterior choroidal artery (red arrow), providing collateral flow to the MCA, which fills in a delayed fashion (black dotted arrow). Early arterial phase $(C)$ demonstrates deep collaterals from the anterior choroidal artery (red arrow), providing collateral flow to the MCA, which fills in a delayed fashion (black dotted arrow). Late arterial phase $(D)$ shows leptomeningeal collaterals (red dotted arrows) arising from branches of the anterior choroidal artery (white arrow), providing collateral flow to the distal portion of the pericallosal artery (blue arrow), which fills in a delayed fashion. Also visible are leptomeningeal collaterals (red dotted arrows) arising from posterior cerebral artery (white dotted arrow), supplying the distal portion of the callosomarginal artery (blue dotted arrow).
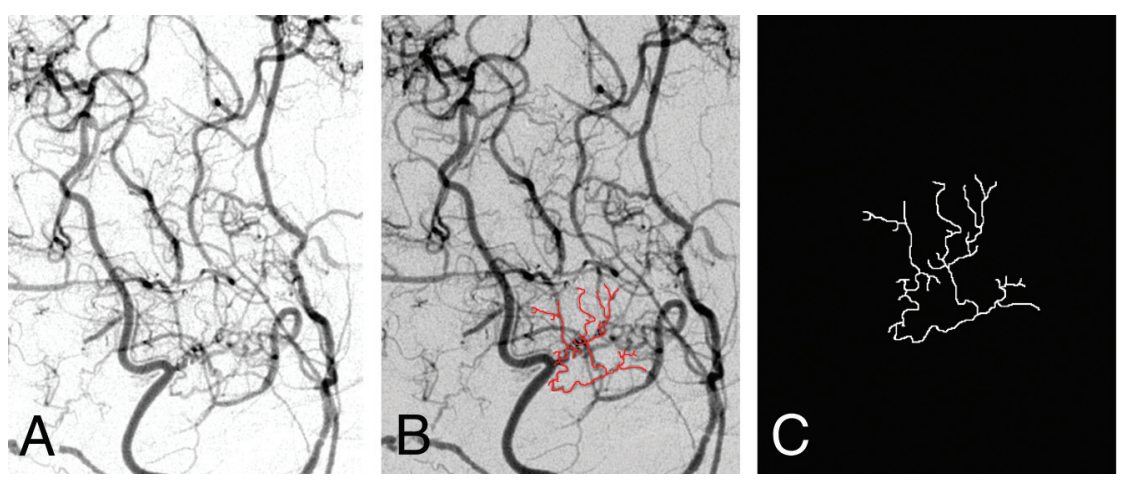

FIG 3. Isolation of collaterals by dynamic delineation. Selected vessels are followed through the arterial phase of the digital subtraction angiograms to establish continuity of vessels. Vessels are traced and marked, excluding other overlapping or underlying vessels; then they are converted to a binary black and white image. $A$, Selective postoperative lateral external carotid artery injection. B, Branches of the superficial temporal artery traced and marked. C, Converted binary image. which is flow-dependent and hypoxiaindependent. In arteriogenesis, preexisting vessels with stenotic segments are exposed to increased shear stress due to a high-pressure gradient between 2 vascular territories. ${ }^{6,22}$ Arteriogenesis involves the recruitment and enlargement of preexisting vessels, leading to the formation of large tortuous vessels. Because sprouting and splitting are not part of this process, it is expected that vessels formed by arteriogenesis will be of lower complexity than those formed by angiogenesis.

Our study shows that there is a distinct difference in angioarchitecture between postsurgical EDAS collaterals and innate collaterals. These differences were detected by analyzing 3 different aspects of the morphology of the collaterals: the branching pattern, tortuosity, and fractal connectivity. While these have been previously established in the description of vascular morphology in the systemic circulation, ${ }^{9,10,27,28}$ this is the first study to use all 3 indices in tandem to differentiate and characterize cerebrovasculature. The branching angioscore has previously been used as a marker for angiogenesis. ${ }^{9}$ The tortuosity index has proved to be useful in analyzing coronary arteries to differentiate patients with chronic pressure and volume overload and as a marker of adverse outcomes in connective tissue disorders. ${ }^{10,27,28}$ Local connected fractal dimensions have been used previously to compare differences in retinal vasculature and to differentiate oral epithelium according to the degree of malignancy. $^{29,30}$

Imaging study of the angioarchitecture of cerebral vasculature poses a challenge due to the $3 \mathrm{D}$ orientation of intracranial vessels. While $3 \mathrm{D}$ imaging such as CTA and MRA can provide adequate representation of the angioarchitecture, these imaging modalities have a limited resolution for the evaluation of new collaterals. ${ }^{31,32}$ For optimal resolution, we selected conventional angiography, which has a spatial resolution of 200 $\mu \mathrm{m}$. However, catheter angiograms have the potential limitation of obscuring the true angioarchitecture of cerebral vessels due to overlapping vasculature when projected on a 2D image. By obtaining selective external carotid ar- 

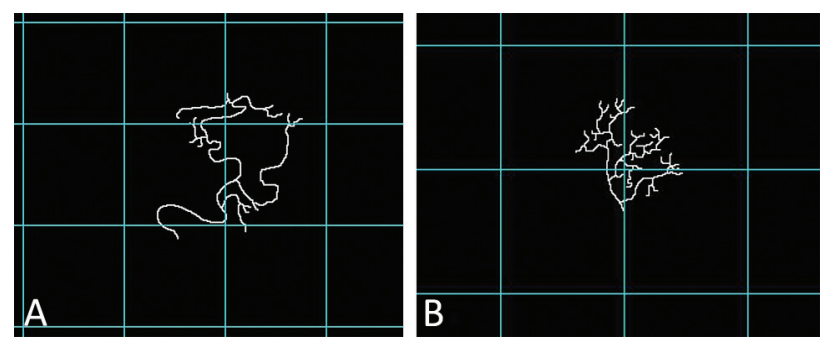

FIG 4. A 10,000-pixel-per-box grid is overlaid on the delineated vessels. The branching angioscore is defined as the total number of branching points within a single box. This is measured in all boxes within the grid containing portions of the delineated vessel, and the box with the highest branching angioscore is selected (highlighted in red). A, Innate collaterals. $B$, EDAS collaterals.
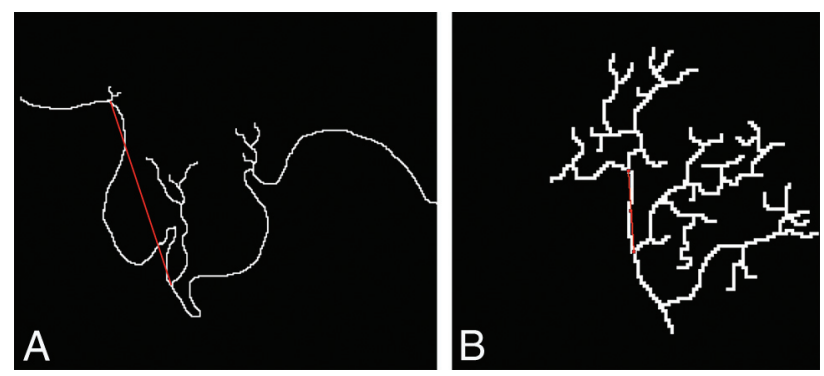

FIG 5. The artery tortuosity index is measured by using the longest branch of the delineated vessel between 2 branching points. The artery tortuosity index is calculated by the quotient of the actual vessel length and the straight-line distance (red line) of a delineated vessel between 2 branching points. A, Innate collaterals. B, EDAS collaterals.

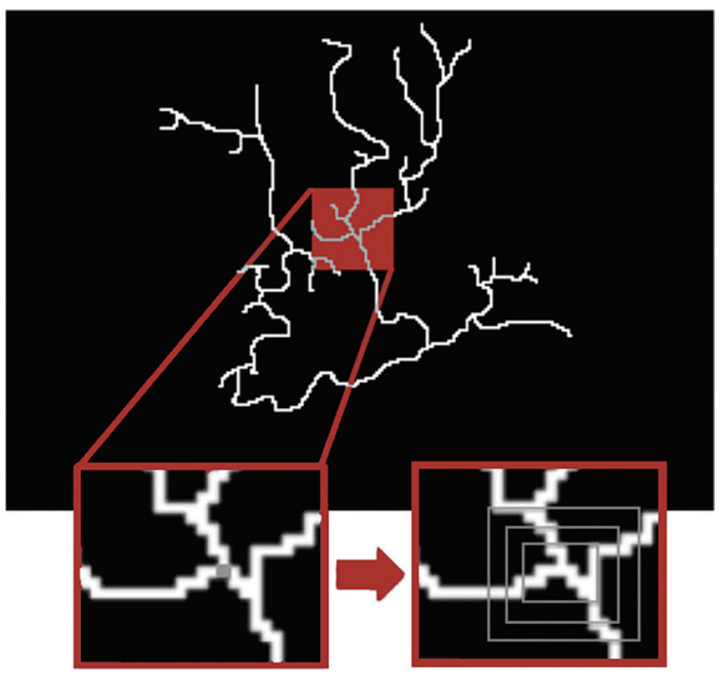

FIG 6. Local connected fractal dimension of delineated vessels. The Fraclac plugin for ImageJ selects seed pixels and measures the total number of connected pixels within a predetermined square area. The process is repeated for increasing concentric areas, and the rate of change of connected pixels is used to calculate the LCFD. The process is repeated for each pixel of the delineated vessel. High connectivity is defined as LCFD $\geq 1.2$.

tery and ICA images, we were able to minimize vessel overlap. Furthermore, dynamic delineation allowed us to isolate targeted vessels for analysis by observing the sequential filling of vessels with time, and by tracing these, we established the continuity of the individual vessels. This method is subject to interobserver
Branching angioscore, tortuosity index, and local connected fractal dimension' by collateral type

\begin{tabular}{lcc}
\hline & \multicolumn{2}{c}{$\begin{array}{c}\text { Type of } \\
\text { Collateral }\end{array}$} \\
\cline { 2 - 3 } & Innate & EDAS \\
\hline No. & 51 & 51 \\
Branching angioscore & & \\
$\quad$ Mean & 4.61 & 14.12 \\
SD & 2.40 & 5.27 \\
Median & 4 & 13 \\
IQR & 3 & 9 \\
Tortuosity index & & \\
Mean & 1.81 & 1.09 \\
SD & 0.66 & 0.07 \\
Median & 1.76 & 1.08 \\
IQR & 0.71 & 0.07 \\
Peak LCFD' & & \\
Mean & 1.16 & 1.28 \\
SD & 0.11 & 0.1 \\
Median & 1.16 & 1.27 \\
IQR & 0.17 & 0.12 \\
\hline
\end{tabular}

Note:-IQR indicates interquartile range.

variability; however, observations were validated internally with good interobserver reliability.

Our study shows that innate collaterals in patients with ICASD display the characteristic high tortuosity and low branching seen in arteriogenesis, affirming the hypothesis that spontaneously occurring collaterals in the brain occur via a hypoxia-independent process and rely on preexisting networks of vessels. In contrast, postsurgical EDAS collaterals have a significantly higher vascular complexity and branching rate. This feature is consistent with the complex branching pattern observed with the formation of new vessels through sprouting and splitting from a parent vessel via angiogenesis. The results of our study support arteriogenesis as the primary mechanism of innate collateral vessel formation within cerebral vasculature in the setting of intracranial arterial stenosis. Our results also suggest that the process of angiogenesis occurs in adult patients with ICASD, leading to the formation of new collateral vessels after EDAS.

Despite the encouraging findings, further studies are necessary to determine whether the postsurgical EDAS vessels are newly formed or correspond to the integration of native vascular networks through new small connections from the external carotid artery. Both scenarios could lead to the same pattern of high LCFD and branching angioscore because the same process forms the vessels of the brain. The low tortuosity index seen in postsurgical collaterals suggests that despite the gradient pressure, these vessels do not become tortuous as seen in native collaterals. This finding may indicate that these vessels do not have high shear stress. Reduced tortuosity and shear stress could be in associated with the absence of hemorrhages from EDAS collaterals as shown in our prior work after up to 7 years of follow-up. ${ }^{4}$

\section{CONCLUSIONS}

The higher complexity and branching rate of collateral vessels formed after EDAS, indicative of sprouting and splitting from a parent vessel, suggest angiogenesis as the primary mechanism of EDAS collateralization. The lower complexity and branching rate and the higher tortuosity index of innate collaterals in patients with ICASD, consistent with large tortuous vessels, suggest 
that arteriogenesis is the primary mechanism for innate collateralization.

Disclosures: Nestor R. Gonzalez—RELATED: Grant: Ruth and Raymond Stotter Endowed Chair in Neurosurgery and the National Institutes of Health-National Institute of Neurological Disorders and Stroke award, K23NS079477. Comments: Research reported in this publication was supported by the National Institute of Neurological Disorders and Stroke of the National Institutes of Health under award No. K23NS079477. The content is solely the responsibility of the authors and does not necessarily represent the official views of the National Institutes of Health.

\section{REFERENCES}

1. Gorelick PB, Wong KS, Bae HJ, et al. Large artery intracranial occlusive disease: a large worldwide burden but a relatively neglected frontier. Stroke 2008;39:2396-99 CrossRef Medline

2. Gonzalez NR, Liebeskind DS, Dusick JR, et al. Intracranial arterial stenoses: current viewpoints, novel approaches, and surgical perspectives. Neurosurg Rev 2013;36:175-84; discussion 184-85 CrossRef Medline

3. Liebeskind DS, Cotsonis GA, Saver JL, et al; Warfarin-Aspirin Symptomatic Intracranial Disease (WASID) Investigators. Collaterals dramatically alter stroke risk in intracranial atherosclerosis. Ann Neurol 2011;69:963-74 CrossRef Medline

4. Dusick JR, Gonzalez NR, Martin NA. Clinical and angiographic outcomes from indirect revascularization surgery for Moyamoya disease in adults and children: a review of 63 procedures. Neurosurgery 2011;68:34-43; discussion 43 CrossRef Medline

5. Dusick JR, Liebeskind DS, Saver JL, et al. Indirect revascularization for nonmoyamoya intracranial arterial stenoses: clinical and angiographic outcomes. J Neurosurg 2012;117:94-102 CrossRef Medline

6. Schirmer SH, van Nooijen FC, Piek JJ, et al. Stimulation of collateral artery growth: travelling further down the road to clinical application. Heart 2009;95:191-97 CrossRef Medline

7. Saito N, Imai H. Insights on the revascularization mechanism for treatment of Moyamoya disease based on the histopathologic concept of angiogenesis and arteriogenesis. World Neurosurg 2011;75: 204-05 CrossRef Medline

8. Schneider CA, Rasband WS, Eliceiri KW. NIH Image to ImageJ: 25 years of image analysis. Nat Methods 2012;9:671-75 Medline

9. Gowdak LH, Poliakova L, Wang X, et al. Adenovirus-mediated $\operatorname{VEGF}(121)$ gene transfer stimulates angiogenesis in normoperfused skeletal muscle and preserves tissue perfusion after induction of ischemia. Circulation 2000;102:565-71 Medline

10. Morris SA, Orbach DB, Geva T, et al. Increased vertebral artery tortuosity index is associated with adverse outcomes in children and young adults with connective tissue disorders. Circulation 2011;124: 388-96 CrossRef Medline

11. Karperien A. FracLac for Image J. http://rsb.info.nih.gov/ij/plugins/ fraclac/FLHelp/Introduction.htm. Accessed March 30, 2015

12. Sakamoto S, Ohba S, Shibukawa M, et al. Angiographic neovascularization after bypass surgery in Moyamoya disease: our experience at Hiroshima University Hospital. Hiroshima J Med Sci 2007;56: 29-32 Medline

13. Matsushima Y, Aoyagi M, Fukai N, et al. Angiographic demonstration of cerebral revascularization after encephalo-duro-arteriosynangiosis (EDAS) performed on pediatric Moyamoya patients. Bull Tokyo Med Dent Univ 1982;29:7-17 Medline

14. Kim DS, Kang SG, Yoo DS, et al. Surgical results in pediatric Moyamoya disease: angiographic revascularization and the clinical results. Clin Neurol Neurosurg 2007;109:125-31 CrossRef Medline

15. Matsushima Y, Suzuki R, Ohno K, et al. Angiographic revasculariza- tion of the brain after encephaloduroarteriosynangiosis: a case report. Neurosurgery 1987;21:928-34 Medline

16. Demartini Z Jr, Martins RT, Rocha CE, et al. Surgical treatment of Moyamoya disease in children. Arq Neuropsiquiatr 2008;66:276-78 Medline

17. Veeravagu A, Guzman R, Patil CG, et al. Moyamoya disease in pediatric patients: outcomes of neurosurgical interventions. Neurosurg Focus 2008;24:E16 CrossRef Medline

18. O'Donnell TF Jr, Callow AD, Scott G, et al. Ultrasound characteristics of recurrent carotid disease: hypothesis explaining the low incidence of symptomatic recurrence. J Vasc Surg 1985;2:26-41 Medline

19. Yamada I, Matsushima Y, Suzuki S. Childhood Moyamoya disease before and after encephalo-duro-arterio-synangiosis: an angiographic study. Neuroradiology 1992;34:318-22 Medline

20. Scott RM, Smith JL, Robertson RL, et al. Long-term outcome in children with Moyamoya syndrome after cranial revascularization by pial synangiosis. J Neurosurg 2004;100(2 Suppl Pediatrics):142-49 CrossRef Medline

21. Houkin K, Kuroda S, Ishikawa T, et al. Neovascularization (angiogenesis) after revascularization in Moyamoya disease: which technique is most useful for Moyamoya disease? Acta Neurochir (Wien) 2000;142:269-76 Medline

22. Heil M, Eitenmüller I, Schmitz-Rixen T, et al. Arteriogenesis versus angiogenesis: similarities and differences. J Cell Mol Med 2006;10: 45-55 Medline

23. Enholm B, Paavonen K, Ristimäki A, et al. Comparison of VEGF, VEGF-B, VEGF-C and Ang-1 mRNA regulation by serum, growth factors, oncoproteins and hypoxia. Oncogene 1997;14:2475-83 CrossRef Medline

24. Liu Y, Cox SR, Morita T, et al. Hypoxia regulates vascular endothelial growth factor gene expression in endothelial cells: identification of a 5' enhancer. Circ Res 1995;77:638-43 Medline

25. Oh $\mathrm{H}$, Takagi $\mathrm{H}$, Takagi $\mathrm{C}$, et al. The potential angiogenic role of macrophages in the formation of choroidal neovascular membranes. Invest Ophthalmol Vis Sci 1999;40:1891-98 Medline

26. Luo J, Martinez J, Yin X, et al. Hypoxia induces angiogenic factors in brain microvascular endothelial cells. Microvasc Res 2012;83: 138-45 CrossRef Medline

27. Wang Q, Liu C, Yan B, et al. Correlation of extracranial internal carotid artery tortuosity index and intraprocedural complications during carotid artery stenting. Eur Neurol 2012;68:65-72 CrossRef Medline

28. Jakob M, Spasojevic D, Krogmann ON, et al. Tortuosity of coronary arteries in chronic pressure and volume overload. Cathet Cardiovasc Diagn 1996;38:25-31 CrossRef Medline

29. Landini G, Rippin JW. How important is tumour shape? Quantification of the epithelial-connective tissue interface in oral lesions using local connected fractal dimension analysis. J Pathol 1996;179: 210-17 CrossRef Medline

30. Landini G, Murray PI, Misson GP. Local connected fractal dimensions and lacunarity analyses of $\mathbf{6 0}$ degrees fluorescein angiograms. Invest Ophthalmol Vis Sci 1995;36:2749-55 Medline

31. Karamessini MT, Kagadis GC, Petsas T, et al. CT angiography with three-dimensional techniques for the early diagnosis of intracranial aneurysms: comparison with intra-arterial DSA and the surgical findings. Eur J Radiol 2004;49:212-23 CrossRef Medline

32. Green D, Parker D. CTA and MRA: visualization without catheterization. Semin Ultrasound, CT MR 2003;24:185-89 CrossRef Medline 